\title{
SALUD MENTAL COMUNITARIA
}

\author{
Jeannette Méndez \\ Terapeuta Ocupacional U.N. Hospital Vista Hermosa Primer Nivel de Atención \\ Magister en Desarrollo Educativo y Social. UPN
}

Si hablamos de Salud Mental, esta es muy ambigua, por eso parto de la sociedad y la salud para poder pensar en Salud Mental (1) Comunitaria.

La sociedad está siguiendo un rumbo como consecuencia de todos los factores; ambientales, biológicos, políticos, económicos, culturales e históricos, que inciden sobre ella. Este proceso puede ir en dẹterioro, permanecer estancado o cambiar hacia el mejoramiento y el progreso social.

A la base de la sociedad se encuentran los aspectos relacionados con la salud, por lo tanto el concepto de salud que se maneja determina acciones a seguir en pro del desarrollo social.

Dentro de esta perspectiva el concepto de salud integral de Calderón, $H$ (1988) 1 nos plantea que "El manejo de la salud - enfermedad a nivel individual o colectivo no debe verse solo a través de procesos ascépticos y técnicos, sino también teniendo en cuenta los procesos naturales, sociales e históricos, los que hacen más comprensible el fenómeno de la vida, su condición y sus circunstancias y cuya influencia no debe desconocerse en ninguno de sus ámbitos", este concepto nos permite ver nuevas consideraciones de la salud.

El cambio de referentes de la salud definida como ausencia de enfermedad a la salud entendida como una relación de diversos aspectos (físico, mental, psicológico, social) conlleva a que el concepto de Salud Mental integre igualmente los aspectos relacionados con las esferas de desarrollo e interacción del hombre.

La mirada a lo social; donde se entretejen las relaciones de los individuos, donde se crea cultura, donde se vive lo cotidiano, donde se recrea el afecto, nos permite leer como un texto la expresión del individuo y desde allí retomar el sentido y significado de la Salud Mental.

Dentro de este marco se hace la Reestructuración de la Atención Psiquiátrica promulgada a través de la Aclamación de la Declaración de Caracas en Noviembre de 1990, donde declaran: "Que la reestructura-

(1) MINISTERIO DE SALUD: El campo de Salud Mental 
ción de la atención psiquiátrica ligada a la Atención Primaria en Salud y. en el marco de los Sistemas Locales de Salud permite la promoción de modelos alternativos centrados en la comunidad y dentro de sus redes sociales".(Declaración de Caracas).

Como resultado se elaboró un Documento Marco que junto con el apoyo de la OPS, establecía los lineamientos generales para el desarrollo de la Estrategia (Bogotá,1986). Y la elaboraciòn de un proyecto de Atención Primaria en Salud Mental, Alcoholismo y Farmacodepencia.

Este enfoque se ha ido cambiando en los últimos años y es así como la OMS afirma en el documento "The introduction of a Mental Health Component into Primary Health Care $(1990)^{2}$, que dicho componente incluye dos áreas distintas : la primera busca fortalecer el funcionamiento de servicios generales de salud, apoyar el desarrollo socioeconómico, mejorar la calidad de vida y promocionar la salud mental y emocional; a la segunda le concierne el control de enfermedades mentales y neurológicas", en: (La Atención Primaria en Salud, 1992).

Retomaremos cada uno de los componentes; con el primer componente, se busca que la estrategia de atención primaria en Salud Mentral vaya más allá de ser una propuesta alternativa de trabajo en salud. Implica cuestionar las prácticas sociales, las formas de organización, los modelos y valores que repercuten en las interacciones que se establecen para expresar, interpretar y dar respuesta a las necesidades. Lo anterior implica romper con la polaridad salud - enfermedad, individual - comunitario, visto en la Salud Mental, se hace aún más difícil, ya que las prácticas en torno a los fenómenos de desmoralización, violencia, anomia y marginalidad en las comunidades, han sido tratar de patologizarlas para dar como respuesta un tratamiento.

Sin embargo en este campo ha sido importante avance, la introducción del concepto de prevención, que supone intervenir antes de que aparezcan los problemas, precisamente para impedirlos, con base en la determinación de riesgos y vulnerabilidades que deben controlarse. Pero esto es muestra clara de que la enfermedad es aún la actriz protagónica de esta trama y que todos los esfuerzos tienen como fin impedir su presencia.

Como la salud se sigue subordinando a la enfermedad, en lo comunitario se realizan técnicas estereotipadas ${ }^{2}$ de prevención reducidas al "informar y educar" en campos de aplicación específica como vacunación, etc. 
En Salud Mental, debemos entonces considerar otro concepto, que al conjugarse con la prevención nos permitia superar el fantasma de la enfermedad. Este podría ser el de Promoción, de acuerdo con la "Carta de Ottawa para la Promoción de la Salud 1986,3 . esta consiste en proporcionar a los pueblos los medios necesarios para mejorar su salud y ejercer un mayor control sobre la misma. Para alcanzar un estado adecuado de bienestar físico, mental y social un individuo o grupo debe ser capaz de identificar y realizar sus aspiraciones, de satisfacer sus necesidades y de cambiar o adaptarse al medio ambiente. La salud se percibe pues, no como el objetivo, sino como la fuente de riqueza de la vida cotidiana".

Por lo anterior el diseño de acciones en la comunidad debe buscar un impacto que favorezca el desarrollo humano de las poblaciones con quienes trabajamos y se reviertan en ellas mismas, es por eso que se requiere que estas vayan dirigidas a grupos poblacionales que tengan puntos de encuentro entre si, donde sus relaciones permitan un crecimiento tanto individual como colectivo.

La familia como base de la organización social es el punto de reunión de los diversos intereses y necesidades. Es a ella a quien considero debe ofrecérsele atención y apoyo para buscar las transformaciones a las que me he referido.

Pero atender el núcleo familiar en su totalidad resulta dificil y lograrlo buscando mayores coberturas aún más, es por ello que la escuela, el colegio, nos proporciona el espacio de referente y convocatoria para ello. Facilitando asi el encuentro de realidades diversas; el niño, el adulto. Es el espacio del acercamiento de la historia de vida que no se quiere repetir, pero que se hace de manera diferente, encontrando como las acciones del pasado, se actuan ahora diferente (maltrato, abuso sexual, violencia intrafamiliar, familias inestables ).

La puesta en común de los interrogantes que cada uno tiene y el escuchar la forma en que algunos le han encontrado solución permite superar el sentido de soledad en los problemas, promoviendo a partir de las experiencias el encuentro de alternativas para solucionar las dificultades que todos y cada uno tienen.

Retomando ahora nuestro segundo componente de la Salud Mental en el cuidado de la Salud Primaria, vemos como este nos refiere a las acciones de rehabilitación. 
A partir de la Declaración de Caracas y con la necesidad de la desinstitucionalización, la Secretaría Distrital de Salud crea los Hospitales Día para la atención de Eñfermôs Mentales Crónicos y Farmacodependientes, esta modalidad de atención busca ser un paso intermedio entre la hospitalización en crisis y la reinserción familiar, social y laboral.

Desde allí hemos observado como las acciones intrahospitalarias con la persona con Enfermedad Mental Crónica y su familia se quedan cortas en el proceso de reinserción social, debido a que las características de enfermedad los han llevado a ser seres aislados o ser considerados como agresivos y peligrosos para sus comunidades, lo cual conlleva un rechazo hacia ellos evitándoles así participar de las actividades barriales.

Por lo anterior debemos retomar para nuestra intervención no solo el núcleo familiar, sino las personas y grupos que hacen parte de la comunidad a la cual pertenecen nuestros usuarios, es decir, participar de la RED SOCIAL de ellos.

Elkaim (1989) desarrolla el concepto de red social como un proceso de construcción permanente tanto individual como colectivo, considerándolo como un sistema abierto y que a través de un intercambio dinámico entre sus integrantes y con integrantes de otros grupos sociales, posibilita la potencialización de los recursos que poseen.

Por lo tanto una red social, se constituye en una fuente esencial de sentimiento de identidad, del deber ser, de competencia, de acción, así como las actitudes habituales a nivel de la salud y del modo de adaptación en caso de crisis.

Es así, como las acciones de promoción a través de redes sociales, debe desarrollarse a partir del concepto de salud, según David Bersh sobre salud, quien concluye "La salud se reconoce implícita o explícitamente, como un fenómeno que presenta variaciones y que sus causas o factores determinantes son múltiples. Porque varía, es decir, porque cambia de un estado a otro es posible tratar de influir en ella". Con esta visión, los aportes que se generan en la intervención comunitaria promueven los estados de salud, ya que parten de las condiciones particulares de cada una de las personas para generar interacciones en un mundo que no este cargado de enfermedad, donde pueda actuar mostrando una cara diferente de sí, donde construya relaciones en las cuales no medie la enfermedad sino el logro.

Én estos procesos de Intervención Comunitaria desde la prevención, la promoción y la rehabilitación está implícita una acción intersectorial, 
porque a la educación, la justicia, la cultura, lo social, el medio ambien-: te y los servicios públicos están implicados en la calidad y el mejốramiento de vida de nuestras comunidades que como lo hemos dicho es salud:

Igualmente como partes de esos diferentes sectores, nos quedamos en acciones interdisciplinarias, brindando una visión segmentada de las. personas y las comunidades, este tipo de prácticas debe tráscender el logro de la transdiciplinariedad que posibilite acciones individuales de los profesionales, que reflejan un trabajo de colectivo pero donde las acciones puntuales y específicas puedan ser atendidas por quien corresponda.

Por lo anterior las estrategias de intervención comunitaria en Salud Mental, deben buscar el potencializar al hombre desde su historia de vida, donde se recupere su bagaje histórico, cultural, los cuales se reflejan en la manera en que se enfrentan a la vida, resuelven conflictos, se relacionan en pareja, familia y construyen comunidad.

Debemos fomentar el desarrollo de talleres que posibiliten la expresión de sentimientos de miedo, alegría, tristeza, rabia, donde se valoren la superación de circunstancias dolorosas particulares, y encontrar que se comparten. valores que son parte de la formación de la vida, y al socializarsen crean un sentido de pertenencia de grupo.

A partir de este encuentro se busca que con la experiencia de unos y las expectativas de los otros se construya una realidad propia donde cada uno sea el actor y el protagonista de sus decisiones. Es propender por hombres más autónomos, capaces de descubrir que ellos tienen la decisión de ser felices, que las palabras calidad de vida tienen un sentido y por ende aportan al progreso social.

En la intervención comunitaria en Salud Mental, encontramos como la palabra adquiere un gran sentido y valor porque es a través de ella que recuperamos la acción del pasado, la entendemos y la transformamos en nuevas acciones.

Es rescatar a través de actividades el valor de la palabra del niño para que sea válida su interlocución con el adulto.

Como Terapeutas Ocupacionales debemos trascender de la acción y darle un valor a la palabra. Cuando nos llega un usuario al consultorio o a la institución le ofrecemos nuestros servicios a cada uno de ellos, sin pensar que hacen parte de un todo del que poca referencia tenemos, les invito a transgredir los límites del consultorio, de la institución y vol- 
teen a ver una realidad que nos muestrá múltiples dificultades que requiere de nuestra acción y que es en ella de donde pertenecen nuestros usuarios.

El trascender es un compromiso ético, profesional, social al cual las seduzco. 


\section{Bibliografía}

-BERSH, D. El fenómeno de la Salud.

- MINISTERIO DE SALÚD, Ėl Campo de la Salud Mental. 1992.

-.-.-, La Atención Primaria. 1992.

- ORGANiZACION MUNDIAL DE LA SALUD, Carta de Ottawa para la Promoción de la Salud, 1986.

-ROTELLI, F. Desinstitucionalización; otra vía. Revista Asociación Especial Neuropsiquiatría. Vol VII No. 21. 1987. 